\title{
Objective and Subjective Physical Activity Levels in Austrian Middle School Students
}

\author{
Carla Greier1, Clemens Drenowatz' ${ }^{2}$, Herbert Riechelmann³, Klaus Greier ${ }^{1,4^{*}}$ \\ ${ }^{1}$ Department of Sport Science, Leopold-Franzens-University of Innsbruck, Innsbruck, Austria \\ ${ }^{2}$ Division of Sport, Physical Activity and Health, University of Education Upper Austria, Linz, Austria \\ ${ }^{3}$ Medical University of Innsbruck, Innsbruck, Austria \\ ${ }^{4}$ Division of Physical Education, Private Educational College (KPH-ES), Stams, Austria \\ Email: *nikolaus.greier@kph-es.at
}

How to cite this paper: Greier, C., Drenowatz, C., Riechelmann, H., \& Greier, K. (2021). Objective and Subjective Physical Activity Levels in Austrian Middle School Students. Advances in Physical Education, $11,448-459$.

https://doi.org/10.4236/ape.2021.114037

Received: September 15, 2021

Accepted: November 23, 2021

Published: November 26, 2021

Copyright $\odot 2021$ by author(s) and Scientific Research Publishing Inc. This work is licensed under the Creative Commons Attribution International License (CC BY 4.0).

http://creativecommons.org/licenses/by/4.0/

\begin{abstract}
Background: Insufficient physical activity is considered a major threat to future public health as many children and adolescents do not engage in the recommended amount of physical activity (PA), despite the well-documented health benefits. The evaluation of ongoing efforts as well as future intervention strategies, however, requires an accurate assessment of PA. Aim: The present study, therefore, examined differences between objectively and subjectively determined PA in 36 Austrian adolescents (52.8\% male; $13.5 \pm 0.5$ years of age). Methods: Time spent in moderate-to-vigorous PA (MVPA) and time spent sedentary was assessed with a wrist-worn accelerometer (GENEActive) that was worn for 7 consecutive days. Self-reported sleep duration was subtracted from total sedentary time in order to obtain sedentary time during waking hours. Participants also completed the short version of the International PA questionnaire (IPAQ) at the end of the objective measurement, which assesses time spent in MVPA and sitting time during the previous week. Results: Objective and subjective data showed that adolescents spent the majority of their time sitting or in sedentary pursuits. Self-reported MVPA was more than double the amount of the objectively determined time spent in MVPA $(p<0.01)$. Accordingly, the majority of adolescents $(88.9 \%)$ met PA recommendations based on self-report, while only $22.2 \%$ were considered sufficiently active when objective PA measurements were used. There were also significant sex differences with higher MVPA as well as lower sedentary time in boys compared to girls $(p<0.01)$. Conclusions: These results highlight the difficulty to accurately determine PA via questionnaire, which may be attributed to irregular PA patterns in youth. Accordingly, subjective measurements may need to be combined with objective methods in order to enhance the accuracy of PA assessments. The low levels of objectively deter-
\end{abstract}


mined PA, particularly in girls, further emphasize the need for continued efforts to ensure an adequate amount of PA in youth.

\section{Keywords}

Adolescents, Moderate-to-Vigorous Physical Activity, Sitting Time, Sedentary Behavior, Accelerometry, Physical Activity Questionnaire

\section{Introduction}

The importance of physical activity (PA) for the development and general well-being of children and adolescents has been well documented (Donnelly et al., 2016; Ortega et al., 2008). Low PA has been associated with poor physical fitness and increased body weight as well as an increased risk for cardio-vascular and metabolic diseases (Kyu et al., 2016; Lee et al., 2012). Given the associated costs, physical inactivity is also a significant economic burden; in Germany, for example, $5 \%$ of the total health care costs are attributed to physical inactivity (CEBR, 2015) and Lee et al. (2017) argue that US \$ 16.6 billion in direct medical costs and US \$ 23.6 billion in lost productivity could be avoided if $75 \%$ of children in the US would exercise 3 times a week. Nevertheless, a majority of children and adolescents are insufficiently active and their behavioral pattern has been characterized by a predominantly sedentary lifestyle (Finger et al., 2018; Tremblay et al., 2016; Van Hecke et al., 2016; Guthold et al., 2020). It is also noteworthy that recent data shows higher levels of insufficient PA in low-income countries compared to high income countries (Guthold et al., 2020). Nevertheless, German data also shows that less than $20 \%$ of 11 - to 17 -year-old adolescents meet current PA recommendations of 60 minutes of moderate-to-vigorous PA (MVPA) (26), which is similar to global levels of insufficient PA of $81 . \%$ (Guthold et al., 2020).

Given the health implications of PA, an accurate assessment of PA is necessary to accurately evaluate the efficacy of various PA intervention strategies along with the association of PA and specific health outcomes (Müller, Winter, \& Rosenbaum, 2010). Even though PA is defined as any bodily movement that increases energy expenditure (Caspersen, Powell, \& Christenson, 1985), it reflects a multi-dimensional behavior that is characterized by duration, intensity and type (e.g., endurance vs. Strength activities) (Bull et al., 2020). The complexity also makes an accurate assessment of PA in its totality a challenging task (Prince et al., 2008). Subjective methods, such as questionnaires or diaries as well as objective methods that include pedometers, accelerometers and a combination of physiological measurements (e.g., heart rate) with accelerometry are commonly used to determine PA in various populations and settings (Müller et al., 2010). At this time there is, however, no single method that can precisely measure all aspects of PA in a natural setting and both objective and subjective measurements remain widely used in PA research. Despite the benefits of objective PA 
assessments, there exist different protocols regarding placement and data processing, which could affect comparability of results (Migueles et al., 2019; Poitras et al., 2016). Aguilar-Farias et al. (2021) further pointed out that there remains a limited understanding of the exchangeability between objective and subjective PA data across different countries.

The present study, therefore, examined the differences in self-reported and objectively determined PA levels in Austrian adolescents. Specifically, differences in MVPA are explored as current PA guidelines focus predominantly on MVPA (Janssen, 2007; Piercy et al., 2018). In addition, differences in time spent sedentary were examined due to the independent association of excess sedentary time with various health outcomes (Owen et al., 2010).

\section{Materials and Methods}

Two middle schools in the Federal State of Tyrol, Austria, were randomly selected for participation. Due to the limited number of accelerometers available only students from $8^{\text {th }}$ grade were included in the study, which resulted in 92 eligible participants between 13 and 14 years of age. Parents provided written informed consent and oral assent was obtained from participants prior to data collection. The study protocol was approved by the Institutional Review Board of the University of Innsbruck, the Tyrolean school board and the participating schools.

Data collection occurred in Mai 2017. The participant's body weight and height were measured according to standard procedures during a school visit with children wearing gym clothes and barefoot. Specifically, body weight was measured to the nearest $0.1 \mathrm{~kg}$ with a calibrated digital scale $\left(\mathrm{SECA}^{\oplus} 803\right.$, Hamburg, Germany) and height was measured with a portable stadiometer (SECA ${ }^{\oplus} 217$, Hamburg, Germany) to the nearest $0.1 \mathrm{~cm}$. Subsequently, body mass index (BMI) was calculated $\left(\mathrm{kg} / \mathrm{m}^{2}\right)$ and converted to BMI percentile based on German reference values with the $90^{\text {th }}$ percentile as cutpoint for overweight/obesity (Kromeyer-Hauschild et al., 2001).

\subsection{Objective Assessment}

PA was assessed via a wrist-worn accelerometer (ACC), which also includes a light and temperature sensor (GeneActiv, Activinsights ${ }^{\circledast}$, Kimbolton, UK). The device has been shown to provide valid and reliable information (Esliger et al., 2011; Pavey et al., 2016). Participants wore the accelerometer continuously for one week on the left arm. This was the non-dominant arm of all participants, which has been shown to provide more accurate results when wrist-based accelerometry is used (Montoye et al., 2016). Data was collected at a frequency of 10 $\mathrm{Hz}$ with a 10 second EPOC length. Time spent in MVPA was determined via the accompanying software (GENEActiv, version: 2.2, ActivInsights Ltd. 2010) (Schaefer et al., 2014). As participants also reported the time they went to bed and got up in the morning sedentary time was calculated as total recorded sedentary time minus sleep time. Daily time spent sedentary and in MVPA was subsequently av- 
eraged over the entire week for the comparison with the subjective PA assessment.

\subsection{Subjective Assessment}

PA questions were based on the International Physical Activity Questionnaire Short Form (IPAQ-SF), which is a commonly used assessment tool to determine PA and sitting time over a period of one week (Craig et al., 2003). Specifically, participants reported the number of days they engaged in moderate PA (MPA) and vigorous PA (VPA) as well as walking and the average amount of time spent in these activities per day they engaged in during the previous week. Total time spent in MPA, VPA and walking were subsequently calulated (number of days $\mathrm{x}$ daily PA time) and divided by 7 to obtain average daily values. MVPA was calulated as the sum of MPA and VPA. In addition, daily sitting time during the previous week was reported (Maddison et al., 2007). Participants completed the questionnaire when the accelerometer was returned; accordingly, the subjective report fell in the same time as the objective measurement.

\subsection{Statistical Analyses}

Data was checked for normal distribution and values are reported as means with SD for the total sample and separately for boys and girls, unless prevalence is shown. The association between IPAQ and accelerometry data was initially checked via Spearman correlation with the strength of the association being evaluated as strong $\left(r_{s}>0.5\right)$, moderate $\left(0.5 \geq r_{s} \geq 0.3\right)$ or weak $\left(0.3>r_{s}>0.1\right)$ for positive and negative correlation coefficients (Cohen, 1988). Additionally, differences between objectively determined and self-reported time spent in MVPA as well as sedentary behavior were examined via Bland-Altman plots and dependent t-tests. Eta squared $\left(\eta^{2}\right)$ was used to determine effect size with values above $0.01,0.06$ and 0.14 being interpreted as small, medium or large effect, respectively (Cohen, 1988). All statistical analyses were calculated with SPSS 26.0 and the significance level was set at $p<0.05$.

\section{Results}

Of the 92 eligible participants 38 did not provide parental consent, which resulted in a sample of 54 adolescents for data collection. Two participants reported technical difficulties and 16 students did not wear the armband consistently. Accordingly, valid data was provided by 36 participants ( $47.2 \%$ female), which were included in the analyses. There were no differences in age, body weight and BMIPCT between boys and girls. Boys, however, were significantly taller than girls. Boys also displayed higher PA levels than girls. Objective sedentary time was higher in girls compared to boys but there was no sex difference in subjective sitting time (Table 1).

There was a moderate correlation between IPAQ and ACC measurements for MVPA $\left(r_{s}=0.454, p<0.01\right)$ while there was no significant association between 
reported sitting time and objectively measured sedentary time. Dependent t-tests further showed significantly higher self-reported MVPA compared to MVPA based on ACC $(p<0.01)$ with medium effect size $\left(\eta^{2}=0.07\right)$. The difference of $56.5 \pm 29.7 \mathrm{~min} /$ day in MVPA, however, reflects more than double the amount of self-reported MVPA compared to objectively measured MVPA. Self-reported sitting time, on the other hand, was $186.2 \pm 117.9 \mathrm{~min} /$ day lower than objectively measured total sedentary time $\left(p<0.01, \eta^{2}=0.08\right)$, which reflects a difference of $75 \%$ (Figure 1).

Table 1. Descriptive characteristics for the total sample and separately for boys and girls. Values are mean \pm SD.

\begin{tabular}{cccc}
\hline & $\begin{array}{c}\text { Total Sample } \\
\mathrm{N}=\mathbf{3 6}\end{array}$ & $\begin{array}{c}\text { Girls only } \\
\mathrm{N}=\mathbf{1 7}\end{array}$ & $\begin{array}{c}\text { Boys only } \\
\mathrm{N}=19\end{array}$ \\
\hline Age (years) & $13.5 \pm 0.5$ & $13.5 \pm 0.5$ & $13.5 \pm 0.5$ \\
Height $(\mathrm{cm})^{\star *}$ & $167.3 \pm 6.4$ & $164.1 \pm 5.3$ & $170.2 \pm 6.0$ \\
Weight $(\mathrm{kg})$ & $54.2 \pm 7.6$ & $53.3 \pm 9.3$ & $55.0 \pm 5.7$ \\
BMI percentile & $49.5 \pm 24.9$ & $50.9 \pm 30.1$ & $48.3 \pm 20.0$ \\
IPAQ Sitting $(\mathrm{min} / \mathrm{d})$ & $565.0 \pm 73.3$ & $573.5 \pm 86.7$ & $557.4 \pm 60.3$ \\
IPAQ MVPA $(\mathrm{min} / \mathrm{d})^{\star *}$ & $105.7 \pm 35.0$ & $82.6 \pm 25.5$ & $126.4 \pm 29.2$ \\
ACC sedentary $(\mathrm{min} / \mathrm{d})^{\star *}$ & $751.2 \pm 91.0$ & $796.4 \pm 88.6$ & $710.8 \pm 73.8$ \\
ACC MVPA $(\mathrm{min} / \mathrm{d})^{\star *}$ & $49.2 \pm 15.7$ & $39.8 \pm 13.3$ & $57.7 \pm 12.9$ \\
\hline
\end{tabular}

ACC: accelerometer measured intensities; IPAQ: self-reported behavior; MVPA: moderate-to-vigorous physical activity; ${ }^{\star *}$ significant sex difference, $p<0.01$.

(a)

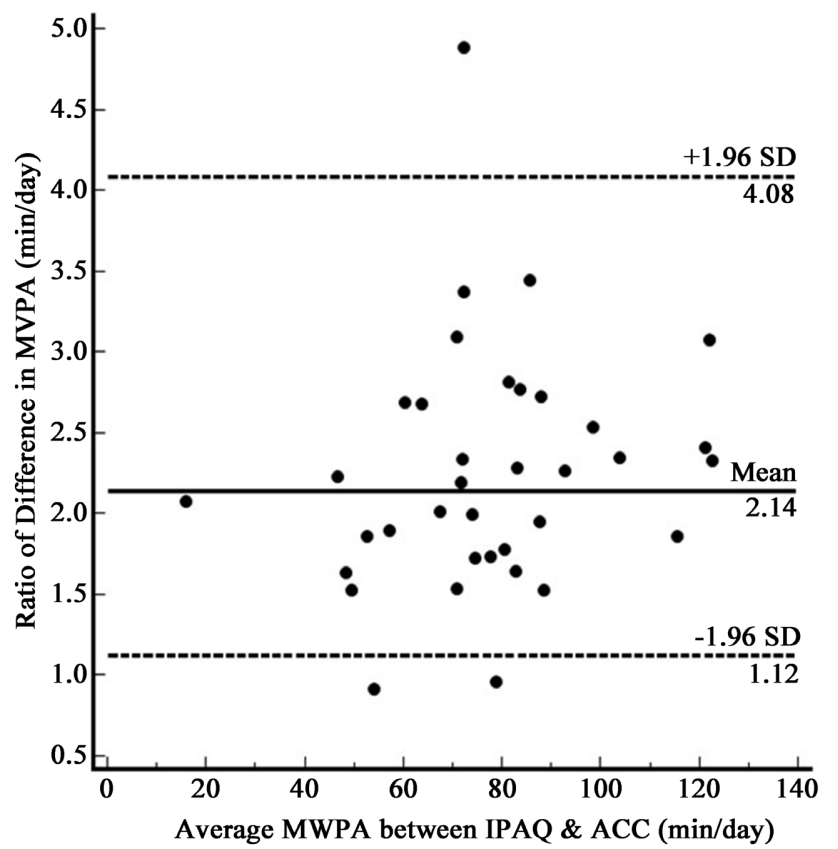

(b)

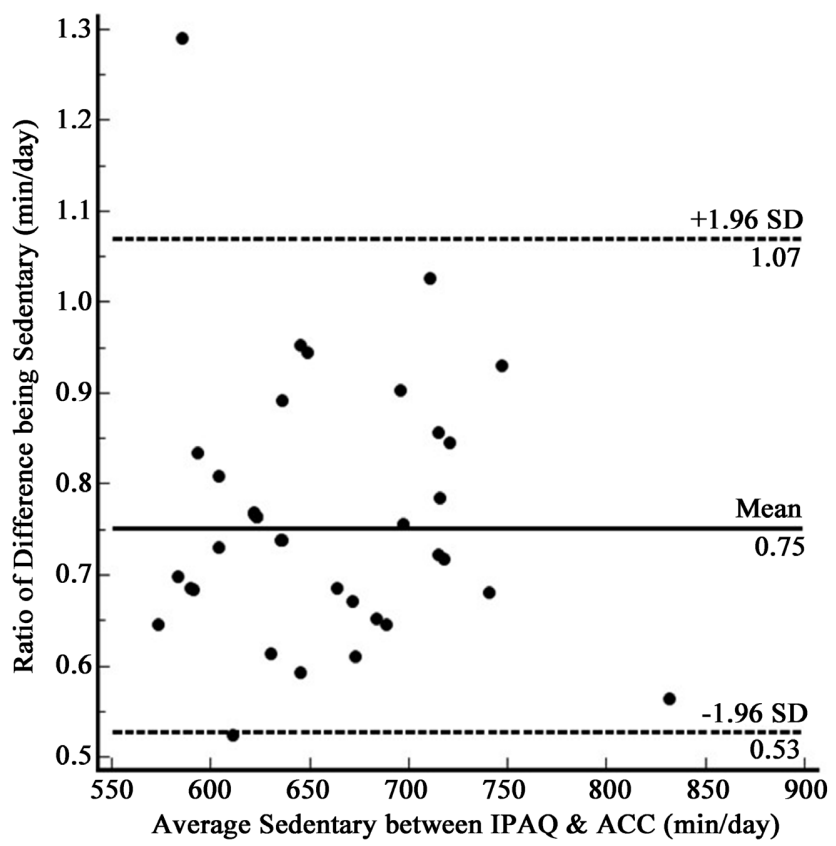

Figure 1. Bland-Altman-Plot showing the ratio of the difference between IPAQ and accelerometry (mean and limits of agreement) for MVPA (a) and sedentary time (b). 
These differences were also reflected by the number of participants considered sufficiently active. Based on IPAQ $88.9 \%$ of the participants (100\% boys, $76.5 \%$ girls) met current PA guidelines of $60 \mathrm{~min} /$ day of MVPA while only $22.2 \%$ (42.1\% boys, no girls) were considered sufficiently active based on the objective assessment of MVPA.

\section{Discussion}

The present study examined MVPA and sedentary behavior via accelerometry and by questionnaire in 13- to 14-year-old middle school students. Independent of the measurement being used, the participants spent the majority of their time in sedentary pursuits and PA levels were lower in girls compared to boys. Self-reported PA levels, however, were significantly higher than accelerometry-based PA. In fact, participants reported more than double the amount of objectively determined PA, which was also reflected by the prevalence of participants being considered sufficiently active. Based on self-report almost $90 \%$ of the participants would have been considered sufficiently active while less than $25 \%$ of participants were meeting PA recommendations of 60 minutes/day when MVPA was assessed via accelerometry. Self-reported sitting time, on the other hand, was lower than accelerometer-determined sedentary time. Given the wide-spread use of questionnaires, particularly in epidemiological studies it is, therefore, possible that current estimations of PA in youth are higher than is actually the case.

The overestimation of PA based on questionnaires has been documented in previous studies (Craig et al., 2003; De Cocker et al., 2007; Gaede-Illig et al., 2014; Lee et al., 2011). Due to the low cost, questionnaires, nevertheless, remain a popular tool in epidemiological studies. Given the important implications of PA for future health (Chaput et al., 2020; Lee et al., 2012), such results can have significant implications for policy decisions. Based on questionnaire data, it could be argued that a majority of Austrian adolescents are sufficiently active and no further efforts are needed to promote PA in youth. Objective data, on the other hand, showed that none of the girls and less than half of the boys are sufficiently active. These results, which are consistent other studies (Hallal et al., 2012; Kettner et al., 2012; Manz et al., 2014), clearly emphasize the need for a stronger commitment to the promotion of PA, particularly in girls. The observed differences in PA between boys and girls are also consistent with previous research (Junger et al., 2018). Sex differences in PA have been attributed to differences in activity preferences and opportunities for engagement in different forms of PA (Vasickova et al., 2013). Further, social aspects need to be considered as the importance of PA among peers is an important correlate of PA (Sterdt et al., 2014; Martins et al., 2017). Additionally, self-efficacy and body image have been addressed as correlates of PA particularly in girls (Nalecz et al., 2012; Sallis et al., 2000). Girls generally show a lower self-efficacy and higher levels of negative body image compared to boys, which has been associated with lower PA and 
higher sedentary time (Añez et al., 2018).

Low PA levels in youth have been commonly attributed to high sedentary time at school and during home work as well as the increasing popularity of electronic devices (e.g., phones, computer) during leisure time (Kaiser-Jovy, Scheu, \& Greier, 2017; Manz et al., 2014). A large amount of youth also rely on motorized transportation to get to school and recreational activities rather than using modes of active transportation.

Accordingly, objective measures of sedentary time exceeded 12 hours/day, with a larger amount of sedentary time in girls compared to boys. A high amount of sedentary time, however, has been associated with various determinetal health effects, independent of PA (Owen et al., 2010). Specifically, sitting time above 8 hours/day, which was exceeded based on IPAQ and accelerometry measurements, has been associated with increased morbidity and mortality (Koster et al., 2012; Patel et al., 2010; Schmid, Ricci, \& Leitzmann, 2015; van der Ploeg et al., 2012) and sitting for more than 10 hours/day was associated with an increased risk for cardiovascular disease (Chomistek et al., 2013). These detrimental effects, however, can be attenuated with engagement in MVPA; according to recent research a minimum of 2.5 minutes of MVPA per hour of sitting time can reduce the increased mortality risk associated with high sitting time in adults (Chastin et al., 2021). As various lifestyle habits are established during adolescence (Gordon-Larsen, Nelson, \& Popkin, 2004) it is critical to implement strategies that reduce sedentary time and increase PA in youth. Intervention efforts should particularly target girls, as they have been shown to spend more time in sedentary pursuits and less time in MVPA (Drenowatz \& Greier, 2019; Hallal et al., 2012).

Some limitations of the present study, however, need to be considered when interpreting the results. A major limitation of the study is the small sample size due to the limited number of accelerometers. In addition, data collection occurred in only two schools and included only students from one grade, which limits the genearlizability of the results. There was also no information on biological maturation, which has been shown to affect PA (Moore et al., 2020). The objective and subjective assessment over the same time period, on the other hand, is a strength of this study.

\section{Conclusion}

Taken together, the results of the present study highlight the difficulties of adolescents to accurately report their PA and sedentary time. This may be attributed to their irregular activity patterns (Sirard \& Pate, 2001). In addition, it should be considered that objective and subjective measurements are not always assessing the same concept (e.g., sedentary time vs. screen time) and, therefore, may not be interchangeable. Rather, objective and subjective measurements such as data from mobile apps, could be used as complementary tools in PA research. Given the low PA levels of adolescents, as shown in this study and previous research 
(Hallal et al., 2012; Manz et al., 2014), it is crucial to obtain accurate data on PA levels in youth. This will allow us to enhance our understanding of correlates of PA in youth and facilitate the development and evaluation of intervention strategies targeting an active lifestyle in adolescents.

\section{Conflicts of Interest}

The authors declare no conflicts of interest regarding the publication of this paper.

\section{References}

Aguilar-Farias, N., Miranda-Marquez, S., Toledo-Vargas, M., \& Chandia-Poblete, D. (2021). Comparison between Self-Reported and Accelerometer-Derived Measurements for Classifying Children and Adolescents as Physically Active in Chile. Cad Saúde Pública, 37, Article ID: e00240620. https://doi.org/10.1590/0102-311x00240620

Añez, E., Fornieles-Deu, A., Fauquet-Ars, J., López-Guimerà, G. Puntí-Vidal, J., \& Sánchez-Carracedo, D. (2018). Body Image Dissatisfaction, Physical Activity and Screen-Time in Spanish Adolescents. Journal of Health Psychology, 23, 36-47. https://doi.org/10.1177/1359105316664134

Bull, F. C., Al-Ansari, S. S., Biddle, S., Borodulin, K., Buman, M. P., Cardon, G., Carty, C. et al. (2020). World Health Organization 2020 Guidelines on Physical Activity and Sedentary Behaviour. British Journal of Sports Medicine, 54, 1451-1462.

Caspersen, C. J., Powell, K. E., \& Christenson, G. M. (1985). Physical Activity, Exercise, and Physical Fitness: Definitions and Distinctions for Health-Related Research. Public Health Reports, 100, 126-131. https://doi.org/10.1136/bjsports-2020-102955

CEBR (Centre for Economics and Business Research) (2015). The Economic Cost of Physical Inactivity in Europe. Centre for Economics and Business Research.

Chaput, J. P., Willumsen, J., Bull, F., Chou, R., Ekelund, U., Firth, J., Jago, R., et al. (2020). WHO 2020 Guidelines on Physical Activity and Sedentary Behaviour for Children and Adolescents Aged 5-17 Years: Summary of the Evidence. International Journal of Behavioral Nutrition and Physical Activity, 17, Article No. 141. https://doi.org/10.1186/s12966-020-01037-z

Chastin, S. F., McGregor D. E., Biddle, S. J., Cardon, G., Chaput, J. P., Dall, P. M., Dempsey, P. C. et al. (2021). Striking the Right Balance: Evidence to Inform Combined Physical Activity and Sedentary Behavior Recommendations. Journal of Physical Activity and Health, 18, 631-637. https://doi.org/10.1123/jpah.2020-0635

Chomistek, A. K., Manson, J. E., Stefanick, M. L., Lu, B., Sands-Lincoln, M., Going, S. B., Garcia, L. et al. (2013). Relationship of Sedentary Behavior and Physical Activity to Incident Cardiovascular Disease: Results from the Women's Health Initiative. Journal of the American College of Cardiology, 61, 2346-2354.

https://doi.org/10.1016/j.jacc.2013.03.031

Cohen, J. (1988). Statistical Power Analysis for the Behavioral Sciences. L Erlbaum Associates.

Craig, C. L., Marshall, A. L., Sjöström, M., Bauman, A. E., Booth, M. L., Ainsworth, B.E., Pratt, M. et al. (2003). International Physical Activity Questionnaire: 12-Country Reliability and Validity. Medicine \& Science in Sports \& Exercise, 35, 1381-1395. https://doi.org/10.1249/01.MSS.0000078924.61453.FB

De Cocker, K., Cardon, G., \& De Bourdeaudhuij, I. (2007). Pedometer-Determined Phys- 
ical Activity and Its Comparison with the International Physical Activity Questionnaire in a Sample of Belgian Adults. Research Quarterly for Exercise and Sport, 78, 429-437. https://doi.org/10.1080/02701367.2007.10599443

Donnelly, J. E., Hillman, C. H., Castelli, D., Etnier, J. L., Lee, S., Tomporowski, P., Lambourne, K., \& Szabo-Reed, A. N. (2016). Physical Activity, Fitness, Cognitive Function, and Academic Achievement in Children: A Systematic Review. Medicine \& Science in Sports \& Exercise, 48, 1197-1222. https://doi.org/10.1249/MSS.0000000000000901

Drenowatz, C., \& Greier, K. (2019). Cross-Sectional and Longitudinal Association of Sports Participation, Media Consumption and Motor Competence in Youth. Scandinavian Journal of Medicine \& Science in Sports, 29, 854-861. https://doi.org/10.1111/sms.13400

Esliger, D. W., Rowlands, A. V., Hurst, T., Catt, M., Murray, P., \& Eston, R. G. (2011). Validation of the GENEA Accelerometer. Medicine \& Science in Sports \& Exercise, 43, 1085-1093. https://doi.org/10.1249/MSS.0b013e31820513be

Finger, J., Varnaccia, G., Bormann, A., Lange, C., \& Mensink, G. (2018). Körperliche Aktivität von Kindern und Jugendlichen in Deutschland. Querschnittergebnisse aus KiGGS Welle 2 und Trends. Journal of Health. Monitoring, 3, 24-31.

Gaede-Illig, C., Zachariae, S., Menzel, C., \& Alfermann, D. (2014). Physical Activity Assessment-A Comparison between IPAQ-SF and SenseWear Pro Armband. Deutsche Zeitschrift für Sportmedizin, 65, 154-159. https://doi.org/10.5960/dzsm.2014.130

Gordon-Larsen, P., Nelson, M. C., \& Popkin, B. M. (2004). Longitudinal Physical Activity and Sedentary Behavior Trends: Adolescence to Adulthood. American Journal of Preventive Medicine, 27, 277-283. https://doi.org/10.1016/j.amepre.2004.07.006

Guthold R et al. (2020). Global Trends in Physical Activity among Adolescents: A Pooled Analysis of 298 Population-Based Surveys with 1.6 Million Participants. Lancet Child \& Adolescent Health, 4, 23-35. https://doi.org/10.1016/S2352-4642(19)30323-2

Hallal, P. C., Andersen, L. B., Bull, F. C., Guthold, R., Haskell, W., Ekelund, U., \& Lancet Physical Activity Series Working Group (2012). Global Physical Activity Levels: Surveillance Progress, Pitfalls, and Prospects. Lancet, 380, 247-257.

https://doi.org/10.1016/S0140-6736(12)60646-1

Janssen, I. (2007). Physical Activity Guidelines for Children and Youth. Canadian Journal of Public Health, 98, 109-121.

Junger, J., Frömel, K., Bergier, J., Salonna, F., \& Niźnikowska, E. (2018). Physical Activity in Students from the Visegrad Countries by BMI Status. Health Problem Civil, 12, 41-48. https://doi.org/10.5114/hpc.2018.74191

Kaiser-Jovy, S., Scheu, A., \& Greier, K. (2017). Media Use, Sports Activities, and Motor Fitness in Childhood and Adolescence. Wiener klinische Wochenschrift volume, 129, 464-471. https://doi.org/10.1007/s00508-017-1216-9

Kettner, S., Wirt, T., Fischbach, N., Kobel, S., Kesztyüs, D., Schreiber, A., \& Drenowatz, C. (2012). Necessity for Physical Activity Promotion in German Children. Deutsche Zeitschrift für Sportmedizin, 63, 94-101. https://doi.org/10.5960/dzsm.2012.016

Koster, A., Caserotti, P., Patel, K. V., Matthews, C. E., Berrigan D., Van Domelen D. R., Brychta R. J. et al. (2012). Association of Sedentary Time with Mortality Independent of Moderate to Vigorous Physical Activity. PLOS ONE, 7, Article ID: e37696. https://doi.org/10.1371/journal.pone.0037696

Kromeyer-Hauschild, K., Wabitsch, M., Kunze, D., Geller, F., Geiß, H., Hesse, V., von Hippel, A. et al. (2001). Perzentile für den Body-mass-Index für das Kindes-und Jugendalter unter Heranziehung verschiedener deutscher Stichproben. Monatsschrift Kinderheilkunde, 149, 807-818. https://doi.org/10.1007/s001120170107 
Kyu, H. H., Bachman, V. F., Alexander, L. T., Mumford, J. E., Afshin, A., Estep, K., Veerman, J. L. et al. (2016). Physical Activity and Risk of Breast Cancer, Colon Cancer, diabetes, Ischemic Heart Disease, and Ischemic Stroke Events: Systematic Review and Dose-Response Meta-Analysis for the Global Burden of Disease Study 2013. BMJ, 354, Article No. i3857. https://doi.org/10.1136/bmj.i3857

Lee, B. Y., Adam, A., Zenkov, E., Hertenstein, D., Ferguson, M.C., Wang, P.I., et al. (2017). Modeling the Economic and Health Impact of Increasing Children's Physical Activity in the United States. Health Affairs, 36, 902-908.

https://doi.org/10.1377/hlthaff.2016.1315

Lee, I. M., Shiroma, E. J., Lobelo, F., Puska, P., Blair, S. N., Katzmarzyk, P. T., \& Lancet Physical Activity Series Working Group (2012). Effect of Physical Inactivity on Major Non-Communicable Diseases Worldwide: An Analysis of Burden of Disease and Life Expectancy. Lancet, 380, 219-229. https://doi.org/10.1016/S0140-6736(12)61031-9

Lee, P. H., Macfarlane, D. J., Lam, T. H. \& Stewart, S. M. (2011). Validity of the International Physical Activity Questionnaire Short Form (IPAQ-SF): A Systematic Review. International Journal of Behavioral Nutrition and Physical Activity, 8, Article No. 115. https://doi.org/10.1186/1479-5868-8-115

Maddison, R., Ni Mhurchu, C., Jiang, Y., Vander Hoorn, S., Rodgers, A., Lawes, C. M., \& Rush, E. (2007). International Physical Activity Questionnaire (IPAQ) and New Zealand Physical Activity Questionnaire (NZPAQ): A Doubly Labelled Water Validation. International Journal of Behavioral Nutrition and Physical Activity, 4, Article No. 62. https://doi.org/10.1186/1479-5868-4-62

Manz, K., Schlack, R., Poethko-Müller, C., Mensink, G., Finger, J., Lampert, T., \& KiGGS Study Group (2014). Physical Activity and Electronic Media Use in Children and Adolescents: Results of the KiGGS Study: First Follow-up (KiGGS Wave 1). Bundesgesundheitsblatt-Gesundheitsforschung-Gesundheitsschutz, 57, 840-848.

https://doi.org/10.1007/s00103-014-1986-4

Martins, J., Marques, A., Peralta, M., Palmeira, A., \& Da Costa, F. (2017). Correlates of Physical Activity in Young People: A Narrative Review of Reviews. Implications for Physical Education Based on a Socio-Ecological Approach. Retos: Nuevas Perspectivas De Educación Física, Deporte Y Recreación, 31, 292-299.

Migueles, J. H., Rowlands, A. V., Huber, F., Sabia, S., \& van Hees, V. T. (2019). GGIR: A Research Community-Driven Open Source R Package for Generating Physical Activity and Sleep Outcomes from Multi-Day Raw Accelerometer Data. Journal for the Measurement of Physical Behaviour, 2, 188-196. https://doi.org/10.1123/jmpb.2018-0063

Montoye, A. H., Pivarnik, J. M., Mudd, L. M., Biswas, S., \& Pfeiffer, K. A. (2016). Validation and Comparison of Accelerometers Worn on the Hip, Thigh, and Wrists for Measuring Physical Activity and Sedentary Behavior. AIMS Public Health, 3, 298-312. https://doi.org/10.3934/publichealth.2016.2.298

Moore, S. A., Cumming, S. P., Balletta, G., Ramage, K., Eisenmann, J. C., Baxter-Jones, A. D., Jackowski, S. A., \& Sherar, L. B. (2020). Exploring the Relationship between Adolescent Biological Maturation, Physical Activity, and Sedentary Behaviour: A Systematic Review and Narrative Synthesis. Annals of Human Biology, 47, 365-383. https://doi.org/10.1080/03014460.2020.1805006

Müller, C., Winter, C., \& Rosenbaum, D. (2010). Current Objective Techniques for Physical Activity Assessment in Comparison with Subjective Methods. Deutsche Zeitschrift für Sportmedizin, 61, 11-18.

Nalecz, H., Guszkowska, M., Mazur, J., \& Dzielska, A. (2012). Self-Efficacy, Self-Esteem and Body Image as Psychological Determinants of 15-Year-Old Adolescents' Physical 
Activity Levels. Human Movement, 13, 264-270.

https://doi.org/10.2478/v10038-012-0031-4

Ortega, F. B., Ruiz, J. R., Castillo, M. J., \& Sjöström, M. (2008). Physical Fitness in Childhood and Adolescence: A Powerful Marker of Health. International Journal of Obesity, 32, 1-11. https://doi.org/10.1038/sj.ijo.0803774

Owen, N., Healy, G., Matthews, C., \& Dunstan, D. (2010). Too Much Sitting: The Population Health Science of Sedentary Behavior. Exercise and Sport Sciences Reviews, 38, 105-113. https://doi.org/10.1097/JES.0b013e3181e373a2

Patel, A. V., Bernstein, L., Deka, A., Feigelson, H. S., Campbell, P. T., Gapstur, S. M., Colditz, G. A., \& Thun, M. J. (2010). Leisure Time Spent Sitting in Relation to Total Mortality in a Prospective Cohort of US Adults. American Journal of Epidemiology, 172, 419-429. https://doi.org/10.1093/aje/kwq155

Pavey, T. G., Gomersall, S. R., Clark, B. K., \& Brown, W. J. (2016). The Validity of the GENEActiv Wrist-Worn Accelerometer for Measuring Adult Sedentary Time in Free Living. Journal of Science and Medicine in Sport, 19, 395-399. https://doi.org/10.1016/j.jsams.2015.04.007

Piercy, K., Troiano, R., Ballard, R. M., Carlson, S. A., Fulton, J. E., Galuska, D. A., George, S., \& Olson, R. (2018). The Physical Activity Guidelines for Americans. JAMA, 320, 2020-2028. https://doi.org/10.1001/jama.2018.14854

Poitras, V. J., Gray, C. E., Borghese, M. M. et al. (2016). Systematic Review of the Relationships between Objectively Measured Physical Activity and Health Indicators in School-Aged Children and Youth. Applied Physiology, Nutrition, and Metabolism, 41, S197-S239. https://doi.org/10.1139/apnm-2015-0663

Prince, S. A., Adamo, K. B., Hamel, M. E., Hardt, J., Gorber, S. C., \& Tremblay, M. A. (2008). Comparison of Direct Versus Self-Report Measures for Assessing Physical Activity in Adults: A Systematic Review. International Journal of Behavioral Nutrition and Physical Activity, 5, Article No. 56. https://doi.org/10.1186/1479-5868-5-56

Sallis, J. F., Prochaska, J. J., \& Taylor, W. C. (2000). A Review of Correlates of Physical Activity of Children and Adolescents. Medicine \& Science in Sports \& Exercise, 32, 963-975. https://doi.org/10.1097/00005768-200005000-00014

Schaefer, C. A., Nigg, C. R., Hill, J. O., Brink, L. A., \& Browning, R. C. (2014). Establishing and Evaluating Wrist Cutpoints for the GENEActiv Accelerometer in Youth. Medicine \& Science in Sports \& Exercise, 46, 826-833.

https://doi.org/10.1249/MSS.0000000000000150

Schmid, D., Ricci, C., \& Leitzmann, M.F. (2015). Associations of Objectively Assessed Physical Activity and Sedentary Time with All-Cause Mortality in US Adults: The NHANES Study. PLoS ONE, 10, Article ID: e0119591. https://doi.org/10.1371/journal.pone.0119591

Sirard, J. R., \& Pate, R. R. (2001). Physical Activity Assessment in Children and Adolescents. Sports Medicine, 31, 439-454.

https://doi.org/10.2165/00007256-200131060-00004

Sterdt, E., Liersch, S., \& Walter, U. (2014). Correlates of Physical Activity of Children and Adolescents: A Systematic Review of Reviews. Health Education Journal, 73, 72-89. https://doi.org/10.1177/0017896912469578

Tremblay, M. S., Barnes, J. D., González, S. A., Katzmarzyk, P. T., Onywera, V. O., Reilly, J. J, Tomkinson, G. R. et al. (2016). Global Matrix 2.0: Report Card Grades on the Physical Activity of Children and Youth Comparing 38 Countries. Journal of Physical Activity and Health, 13, S343-S366. https://doi.org/10.1123/jpah.2016-0594

Van der Ploeg, H. P., Chey, T., Korda, R. J., Banks, E., \& Bauman, A. (2012). Sitting Time 
and All-Cause Mortality Risk in 222,497 Australian Adults. Archives of Internal Medicine, 172, 494-500. https://doi.org/10.1001/archinternmed.2011.2174

Van Hecke, L., Loyen, A., Verloigne, M., van der Ploeg, H. P., Lakerveld, J., Brug, J., De Bourdeaudhuij, I. et al. (2016). Variation in Population Levels of Physical Activity in European Children and Adolescents According to Cross-European Studies: A Systematic Literature Review within DEDIPAC. International Journal of Behavioral Nutrition and Physical Activity, 13, Article No. 70.

https://doi.org/10.1186/s12966-016-0396-4

Vasickova, J., Groffik, D., Frömel, K. et al. (2013). Determining Gender Differences in Adolescent Physical Activity Levels Using IPAQ Long Form and Pedometers. Annas of Agricultural and Environmental Medicine, 20, 749-755. 